\title{
Crystalline lattice effects on tensionless surface dynamics
}

\author{
Esteban Moro*, Rodolfo Cuerno, Angel Sánchez \\ Grupo Interdisciplinar de Sistemas Complicados and Departamento de Matemáticas, c/ Butarque 15, \\ Universidad Carlos III de Madrid, E-28911 Leganés, Madrid, Spain
}

\begin{abstract}
A new model is introduced for two-dimensional crystalline interfaces with negligible surface tension. The model is given by a discrete version of the linear molecular beam epitaxy (MBE) equation plus an additional term periodic in the interface height variable. Langevin dynamics simulations and analytical arguments show that the model exhibits a roughening transition to the high temperature phase of the sine-Gordon model, whose initial stages are nevertheless described by the scaling of the linear MBE equation. Out of equilibrium, the model can have three different behaviors depending on temperature and deviation from equilibrium: A non-moving flat interface, a moving interface with oscillatory roughness, and a moving interface which shows scaling. Possible connections to experiments are discussed.
\end{abstract}

Keywords: Surface growth; Surface diffusion; Roughening transition; Kinetic roughening; Molecular beam epitaxy

\section{Introduction}

Surface and interface roughness are exceedingly important problems in the fabrication of low dimensional semiconductor devices [1,2]. Rough surfaces develop as a consequence of competition among different effects, such as surface tension, surface diffusion, thermal fluctuations, lattice effects, applied forces, and so on. Thus, in equilibrium, a particular surface can be rough under certain conditions and macroscopically "flat" under some other ones, these two regimes being separated in many cases by a roughening transition [1,3]. Out of equilibrium, which is usually the case when growing micro and nanoelectronic devices, surfaces can grow in many different modes, ranging from layer by layer (LBL) to rough growth morphologies: Experimentally, ordered LBL growth is detected as oscillations in the reflection high-energy electron diffraction (RHEED) specular intensity [4], whereas for a growing rough interface the coherence leading to RHEED oscillations is lost.

Among the studies of surface dynamics, systems with negligible surface tension have received a lot of attention lately. Relevant instances of tensionless surfaces are thin films grown by, e.g. thermal evaporation, sputter deposition or Molecular Beam Epitaxy (MBE). Generically, we will refer to these as MBE growth [1,4,5]. Villain [6] and Lai and Das Sarma [7], elaborating on classic works by

${ }^{*}$ Corresponding author. E-mail: emoro@math.uc3m.es 
Herring and Mullins [8,9], proposed that surfaces grown in MBE-like conditions obey relaxation mechanisms (namely, surface diffusion) that locally minimize surface curvature. To linear order this is described by the so called linear MBE equation for surface height $h(\boldsymbol{x}, t)$ :

$\frac{\partial h}{\partial t}=-\kappa \nabla^{4} h+I+\xi(x, t)$,

where $\kappa$ is a constant and $\xi$ is a random fluctuation around the average flux $I$. This equation leads to scale invariant behavior of the growing surface both in time and space [1], which has been recently demostrated in real MBE systems [10,11].

Besides the above relaxation mechanism, there are other processes which break this scale free picture of surface growth. One of them is the Schwoebel effect: a diffusion barrier at step edges which prevents an atom from jumping downward to a terrace, and therefore would generate a rough surface containing mounds, whose typical size provides a length scale [6]. On the other hand, lattice effects can also introduce a characteristic length scale equal to the distance between the surface coarsegrained units. There are several experimental situations in which the Schwoebel effect is absent, such as the dynamics of policrystalline interfaces, or surface growth at high enough temperatures. In this work we will neglect Schwoebel barriers and study the interplay between lattice effects and surface diffusion. In principle at high enough temperatures lattice effects are expected to become negligible so that the surface would evolve according to Eq. (1). However, we will see that lattice effects modify the scaling behavior at high temperatures so that Eq. (1) only holds up to a certain crossover time and therefore is not asymptotic.

The model we propose is hence given by the Hamiltonian

$H=\kappa \sum_{i}\left[\left(\sum_{n, n_{i}} h_{j}\right)-n h_{i}\right]^{2}-V_{0} \sum_{i} \cos \left(h_{i} / a\right)-\sum_{i} I h_{i}$,

where the functions $h_{i}(t)$ give the surface height above site $i$ in a two-dimensional (2D) square $(L \times L)$ lattice, and the sum in the brackets runs over the nearest neighbors of site $i, n=4$ being the lattice coordination number; we emphasize that the values of $h_{i}$ are real numbers not restricted to being integers. The first term in Eq. (2) is a discrete version of the Laplacian squared of the height, which favors small surface curvatures and leads to the biharmonic term $\left(\nabla^{4} h\right)$ in the evolution equations (see Eqs. (3) and (4) below). The second term in Eq. (2) is a weighting function which favors $h_{i}$ to be $2 n \pi a$, with $a$ the lattice spacing. Such a pinning potential was first introduced by Chui and Weeks [12] to study a continuous version of the discrete Gaussian model, which in this way becomes a sine-Gordon (sG) model [13]. Finally, the third term in Eq. (2) explicitly includes non-equilibrium effects through the interaction of the surface with applied driving fields $I$ which represent, e.g., the chemical potential due to the flux of incoming particles in MBE [14].

We study model Eq. (2) by Langevin dynamics simulations, i.e., by integrating the corresponding overdamped equations of motion for $h_{i}$ [rescaled as to have $\kappa=a=V_{0}=1$ ]:

$\frac{\partial h_{i}}{\partial t}=-\nabla_{\mathrm{d}}^{2}\left(\nabla_{\mathrm{d}}^{2} h_{i}\right)-\sin h_{i}+I+\xi_{i}(t)$.

$\nabla_{\mathrm{d}}^{2}$ stands for the discrete Laplacian in the square lattice and $\xi_{i}(t)$ are independent Gaussian white noises of zero mean and $\left\langle\xi_{i}(t) \xi_{j}\left(t^{\prime}\right)\right\rangle=2 T \delta_{i j} \delta\left(t-t^{\prime}\right), T$ being the temperature. Thus, Eq. (3) reproduces the long distance behavior of the continuum equation 
$\frac{\partial h}{\partial t}=-\nabla^{4} h-\sin h+I(x)+\xi(x, t)$,

Langevin dynamics has been very successful in the analysis of related models, such as the discrete $2 \mathrm{D}$ $\mathrm{sG}$ equation [15]: For instance, in [16], the equilibrium roughening temperature was determined, confirming the renormalization group (RG) results of Chui and Weeks [17], whereas in [18] it allowed to explain the system behavior near and far from equilibrium. From the MBE viewpoint, the study we carry out in this paper is interesting, as it is clear that if lattice (epitaxial) effects are important, an equation like Eq. (4) could belong in a general description of crystal growth by MBE [19-21].

The outline of this paper is as follows. In Section 2 we present the simulation results of our model in equilibrium and discuss the existence for Eq. (3) of a scaling behavior different from that of Eq. (1) due to the lattice effects. Section 3 is devoted to non-equilibrium results and the driving-temperature phase diagram. The final section is dedicated to a summary of our main conclusions and possible experimental implications.

\section{Equilibrium behavior}

Our study begins with the main question about the equilibrium $(I=0)$ of our model: The existence or not of a roughening transition. As we know of no previous analytical or numerical results for Eqs. (3) and (4), we recall the main RG ideas as applied to the sG equation $[14,17,22]$ : There exists a roughening temperature $T_{\mathrm{R}}$ above which the energy of a step on the surface becomes zero, because temperature "renormalizes" the sine term, effectively suppresing it at $T_{\mathrm{R}}$; along this process, the lattice potential modifies the effective value of the surface tension coefficient [15]. We find similar features in the equilibrium properties of model Eq. (2). In Fig. 1, we plot results of numerical simulations of Eq. (3) [with $h_{i}(t=0)=0$ ]; specifically, we represent the surface structure factor

$S(\boldsymbol{k}, t) \equiv\langle\hat{h}(\boldsymbol{k}, t) \hat{h}(-\boldsymbol{k}, t)\rangle$,

$\hat{h}(k, t)$ being the Fourier transform of $\left(h_{i}(t)-\overline{h(t)}\right)$, with $\overline{h(t)}$ the mean height. We find that, above $T_{\mathrm{R}} \approx 10, S(k, t)$ scales in the stationary regime in the same fashion as for the linear MBE equation

$S(k, t \rightarrow \infty)=\frac{T}{16 \sin ^{4}(k / 2)}$.

In the inset of Fig. 1 we plot the effective $\alpha$ exponent defined from the relationship

$W_{\text {sat }}(L) \equiv W(L, t \rightarrow \infty) \sim L^{\alpha}$,

where $W_{2}(L, t)=\left\langle(h(\boldsymbol{x}, t)-\overline{h(t)})^{2}\right\rangle$ is the surface roughness. For the linear MBE Eq. (1) we have $\alpha=1$, as occurs in our model for $T \geq 10$. We thus identify $T_{\mathrm{R}}$ as the temperature above which the sine term renormalizes to zero and Eq. (3) effectively behaves as the linear MBE equation. Note also in Fig. 1 that the smaller slope of $S(k, t)$ for small $k$ below $T_{\mathrm{R}}$ indicates the momenta range still dominated by the sine term, which shrinks as $T \rightarrow T_{\mathrm{R}}$. Furthermore, for model Eq. (2) we have carried out [23] a variational calculation (which closely follows that presented in [3] for the sG equation), which leads to a prediction of $T_{\mathrm{R}} \approx 12$, in very good qualitative agreement with Langevin dynamics. For the sake of comparison, in our units the roughening transition for the sG model takes place at $T_{\mathrm{R}}^{\mathrm{sG}}=8 \pi$ 


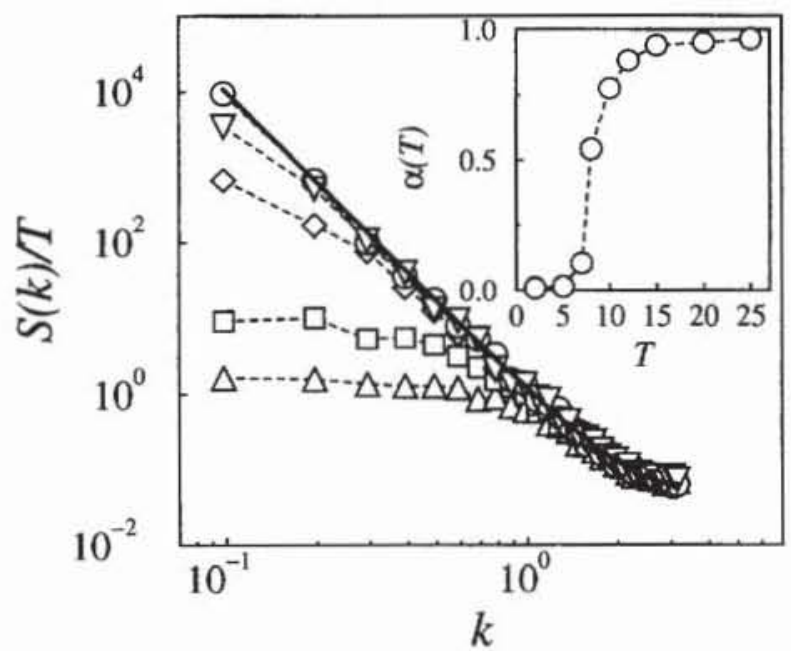

Fig. 1. $S(\mathbf{k}, t)$ vs $k$ in the stationary regime for, bottom to top, $T=2,7,8,10$, and 20 (the last two ones overlap) and $L=64$. Dashed lines are guides to the eye. Solid line is the scaling of the discrete linear MBE equation, $S(\mathbf{k}, t \rightarrow \infty) / T=1 /\left[16 \sin ^{4}\right.$ $(k / 2)$ ]. Inset: Roughness exponent $\alpha$ (defined effectively from $W_{\text {sat }} \sim L^{\alpha}$ ) vs $T$. Error bars are of the symbol size or smaller.

$[16,17,22]$. The difference in the surface profile above and below the roughening temperature is evident in Fig. 2 where we show snapshots of the surface profile at saturation.

After finding $T_{\mathrm{R}}$, we focus on the asymptotic scaling of the high temperature phase. An extension to our model [23] of the RG calculations for the sG model [22] suggests the presence of an effective, possibly small, surface tension term generated by the lattice potential [24]. Actually, we have found that, close enough to $T_{\mathrm{R}}, S(k, t)$ is best fitted assuming that a Laplacian term has been generated. The Laplacian term should dominate the scaling behavior for $T>T_{\mathrm{R}}$ after a crossover time $t_{\times}$(which increases with temperature) observable only for large enough system sizes due to saturation effects. We stress that for $t<t_{\times}$and $T>T_{\mathrm{R}}$, our simulations yield the same dynamics as the linear MBE equation, i.e., $W(L, t) \sim t^{1 / 4}$, whereas for $t>t_{\times}$(and large $L$ ) we have seen evidence for a scaling close to that of the high $T$ phase of the sG equation: $W^{2}(L, t) \sim \log t, W_{\text {sat }}^{2} \sim \log L$, i.e., of Edwards-Wilkinson (EW) type [25]. Although our present computing facilities do not allow us to unambiguously confirm the existence of an asymptotic scaling of EW type (up to $L=512$ ), we obtained further evidence from the average velocity $v$ of an interface given by the transformation $h^{\prime}(x, t)=h(x, t)-\epsilon x^{2}$. In the presence of a finite Laplacian with coefficient $\nu, v$ behaves as $v \sim \nu \epsilon$ when $\epsilon \rightarrow 0$. We have obtained this behavior in our simulations, whose results support a small but distinctly nonzero value for $\nu$, even for temperatures around $T \approx 15$. Further work to settle this question is in progress [23].

\section{Non equilibrium behavior}

For the the non equilibrium $(I \neq 0)$ case, Fig. 3 summarizes our results, which lead to a separation of the $(T, I)$ parameter space into three different regions. In region $A$, the lattice potential controls the dynamics, and the surface shows zero average velocity like in the low $T$ phase at equilibrium. Increasing $I$ further for fixed $T$ within region $A$, a value of the driving is reached which is strong 
(a)

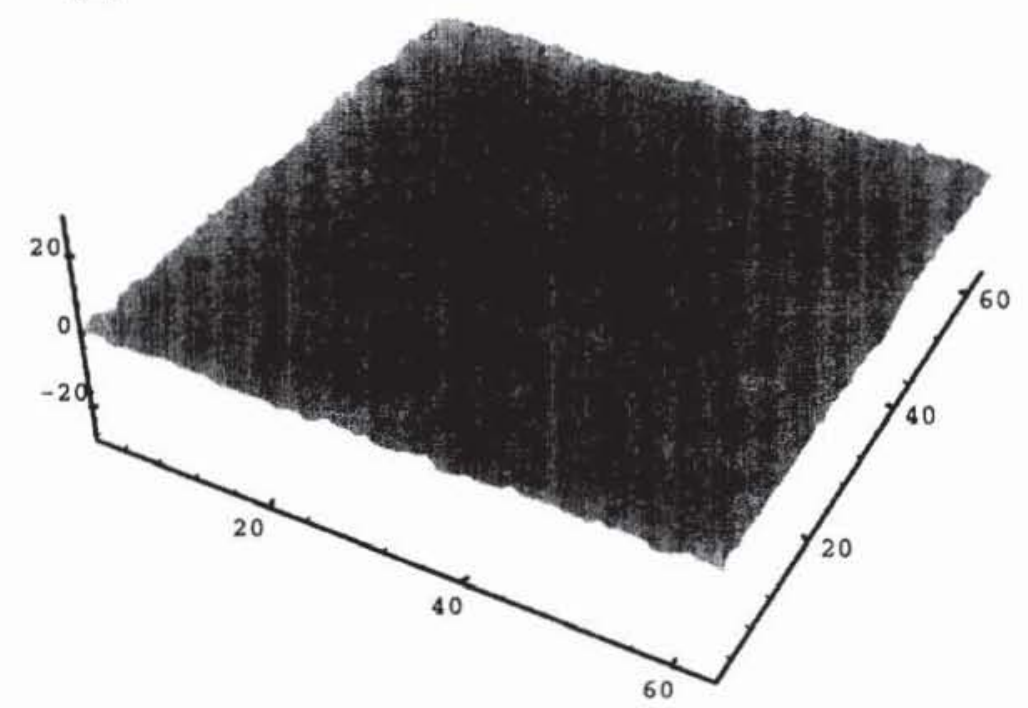

(b)

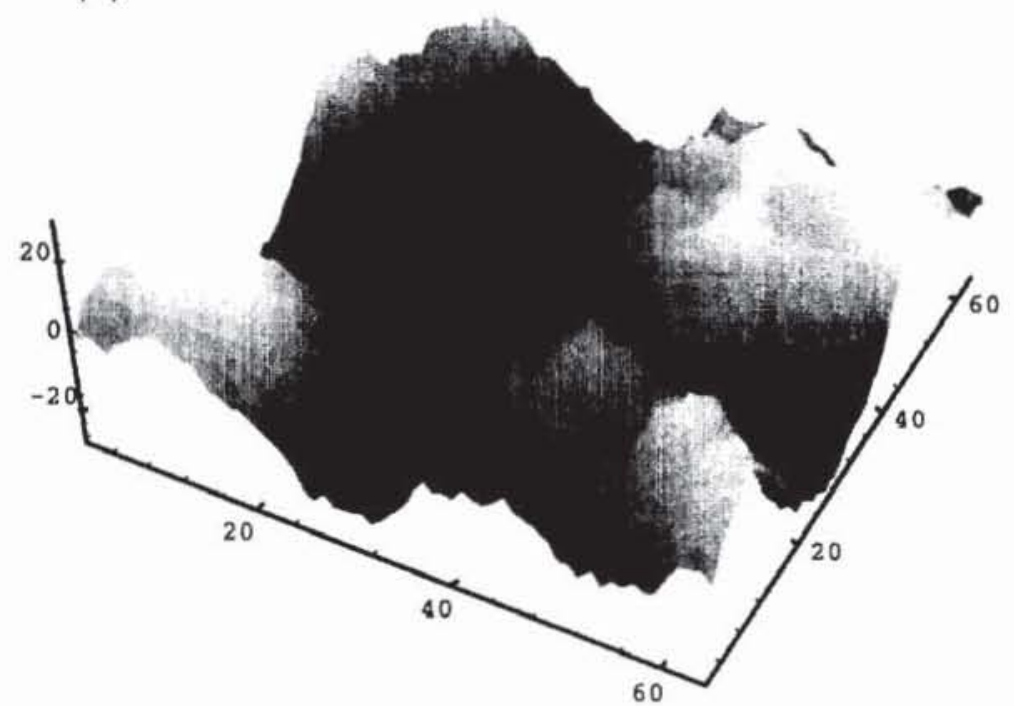

Fig. 2. Surface profiles for temperatures below $\left(T=4\right.$, top) and above $\left(T=12\right.$, bottom) the roughening temperature $\left(T_{\mathrm{R}} \approx 10\right)$ for our model.

enough to pull the surface over the potential barriers, leading to a nonzero average velocity $v$ for the interface; hence the system is in Region $B$. What is more interesting, increasing $T$ for fixed $I$ produces the same effect: This can be appreciated from Fig. 4, where the linear response interface mobility $\mu=v / I$ for $I=0.1$ exhibits a sharp transition from a pinned (region $A$ ) to a moving (region $B$ ) 


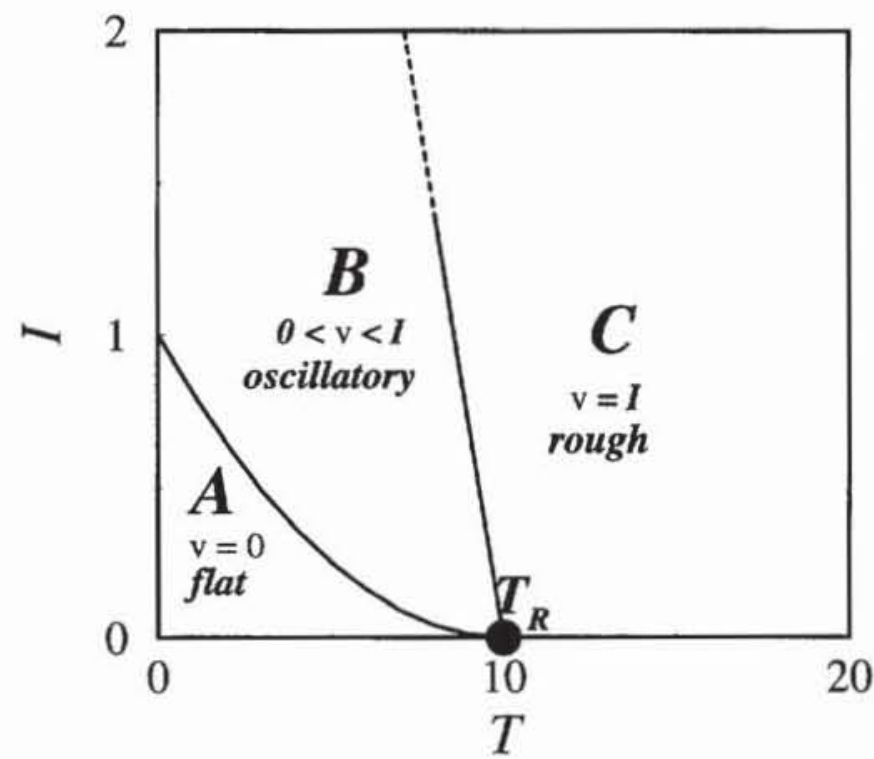

Fig. 3. Sketch of the "phase-diagram" of our model. Lines are approximate and not intended as quantitatively correct, except for the ends of the line separating region $A$ from $B$.

interface. The transition temperature decreases with increasing $F$; in fact, growth occurs for all $T$ when $I \geq 1$, that is, region $A$ terminates at $T=0, I=1$ [26]; this is seen also in Fig. 3 for $I=1.1$.

The difference between regions $B$ and $C$ relates not only to interface mobility but also to

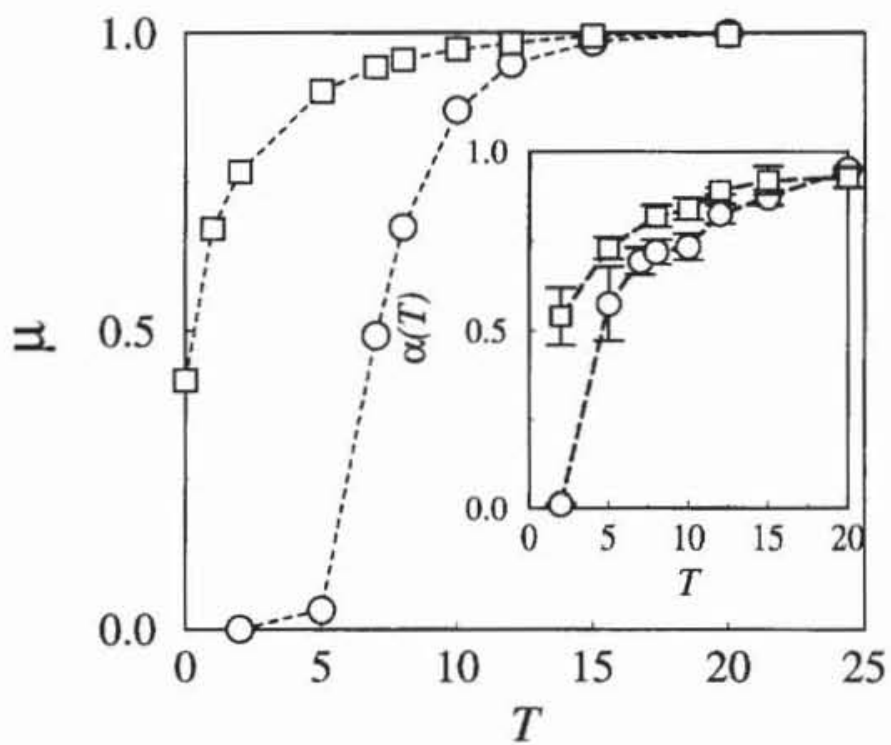

Fig. 4. Linear mobility $\mu \equiv v / l$ vs $T$ for $I=0.1(O)$ and 1.1 ( $\square$ ). Inset: Roughness exponent $\alpha$ vs $T$ for the same values of $I$. Error bars are of the symbol size or smaller except where plotted. 
morphology. In region $C$, the interface mobility is maximum $(\mu \approx 1)$, and the interface displays the same scaling behavior as in the equilibrium high $T$ phase. On the contrary, region $B$ is characterized by a temperature dependent mobility: The interface moves nonlinearly, in a way which is reminiscent of the LBL growth experimentally observed in RHEED oscillations, particulary if one looks at the roughness dependence on time (upper panels of Fig. 5). To understand this behavior, let us recall that, for actual surfaces, there is a competition between surface diffusion, that tends to complete layers before new ones start to form, and the incoming flux, that supplies new material for island nucleation on top of incomplete layers. Consequently, three time scales arise: a surface diffusion time $t_{\mathrm{d}}$ $\left(t_{\mathrm{d}} \sim \kappa^{-1}=1\right.$ in our units), a time associated with the flux rate $t_{\mathrm{r}} \sim I^{-1}$, and a time associated with flux rate fluctuations $t_{\mathrm{T}} \sim T^{-1 / 2}$. As $t_{\mathrm{d}}$ is fixed, the relevant quantity for the dynamics in region $B$ is the ratio $\gamma=t_{\mathrm{T}} / t_{1}$ : Indeed, for large $\gamma$, the height fluctuations due to $T$ grow in a typical time scale $t_{\mathrm{T}}$, much slower than the time needed by the incoming flux to pull the surface over a potential barrier, leading to LBL behavior. On the other hand, for small $\gamma$, temperature fluctuations dominate over the surface growth as a whole, yielding rough, multilayer growth. This is in perfect agreement with what we see in Region $B$, where $\gamma$ decreases when going from Region $A$ to Region $C$. In addition, region $C$ shows no sign of oscillations, one more hint that the potential is effectively renormalized to zero by temperature.

For the purpose of comparison to experiments, we note that roughness oscillations in Region $B$ of our model and RHEED oscillations can be related through the coverage $\theta_{n}$ of the $n$-th layer, i.e., the number of occupied sites in that layer divided by the total number of available sites. The surface roughness reads
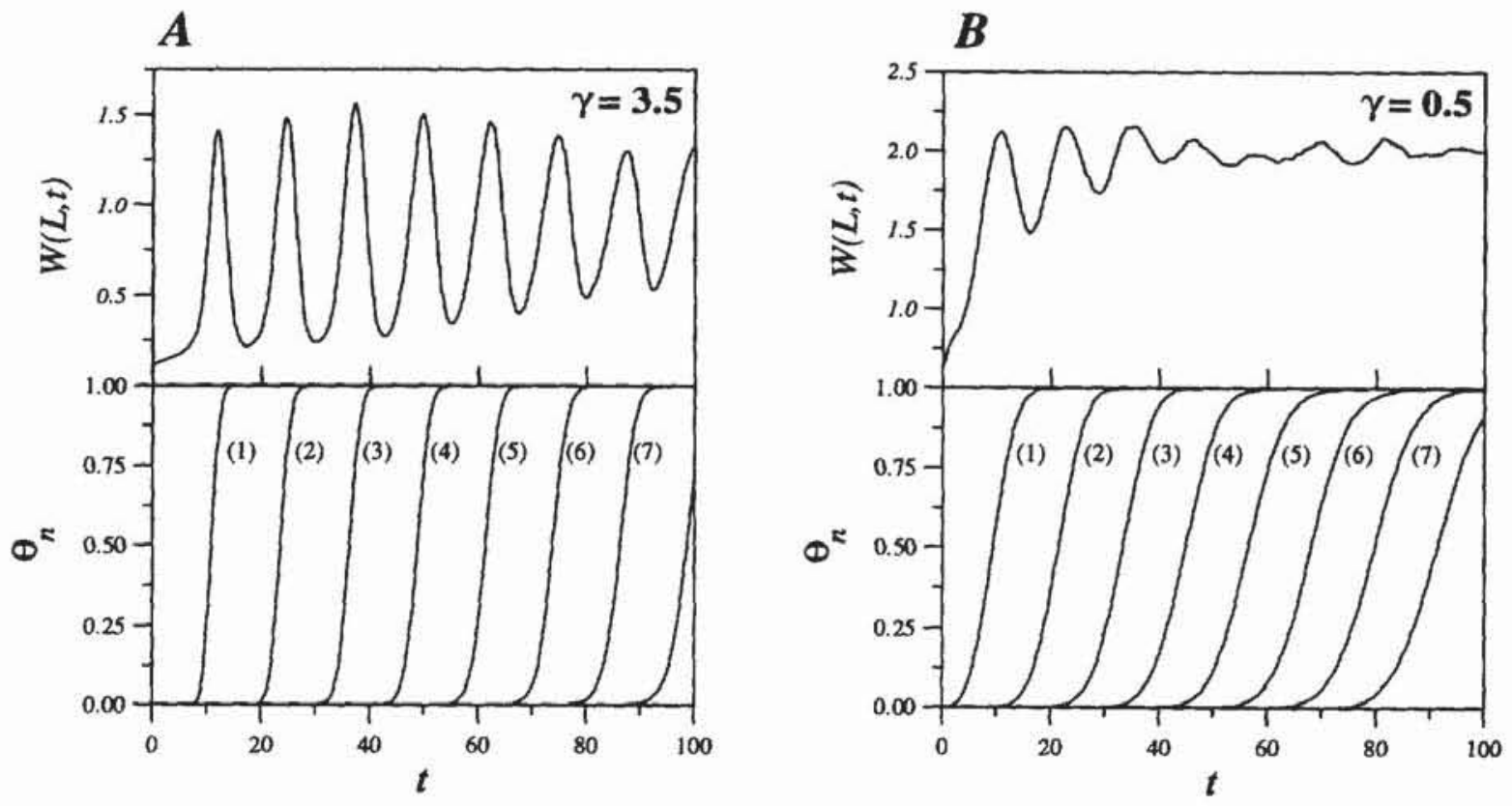

Fig. 5. $W^{2}$ vs $t$ (upper panel) and coverage of the first 7-8 layers vs time (lower panel) for differents values of $I$ and $T$ : $I=0.8$ and $T=3$, i.e., $\gamma=0.46$ for the A plot, and $I=1.1$ and $T=0.1$, i.e., $\gamma=3.5$ fot the B plot. 
$W^{2}=(2 \pi)^{2} \sum_{n}\left(\theta_{n}-\theta_{n+1}\right)(n-\bar{h})^{2}$,

where we have discretized the surface height values: $h_{n}=2 n \pi$. In perfect LBL growth, at any given time $\theta_{n}$ is different from 0 or 1 only for one value of $n$, and $W^{2}=(2 \pi)^{2}\left(\theta_{n}-\theta_{n}{ }^{2}\right)$, oscillating between 0 and $\pi^{2}$ as $\theta_{n}$ goes from 0 to 1. RHEED spectra, which are directly related to coverages [4], behave accordingly, oscillating with constant amplitude in time. On the contrary, when two or more layers are growing at the same time (rough growth), damping terms proportional to $\theta_{i} \theta_{j}$ can be seen to appear in $W^{2}$, and hence oscillations damp out (see Fig. 5), once again as RHEED oscillations do. In all cases, $\gamma$ determines the period of oscillations as well as the damping time. This simple analysis shows that the roughness behavior we see in our model and RHEED oscillations have the same origin and can then be qualitatively related. Region $B$ is thus the parameter range for which one would expect LBL growth (close to region $A$ ) crossing over to rough growth (as one approaches region $C$ ). In region $C$, the suppresion of the lattice potential yields the very notion of layer meaningless.

\section{Conclusion}

We have proposed and studied a model for growth of crystalline tensionless surfaces [Eq. (2)] whose main features are an equilibrium roughening transition with $T_{\mathrm{R}} \approx 10$ (dimensionless units) to a high temperature phase similar to the sG model, and a non-equilibrium "phase diagram" composed of three regions where the surface is pinned, moves nonlinearly, or roughens kinetically. The nonlinear region, $B$, is in fact a crossover region from LBL growth (close to the pinned phase, $A$ ) to rough growth (close to the rough phase, $C$ ). As we have discussed, this characterization is relevant to experiment and real systems may exhibit measurable signatures of the three regimes. We note that, among those usually studied in the context of rough surfaces, no other continuum model without lattice effects has reproduced these oscillations [1]. Presently, reports of RHEED oscillations dependence on temperature are available and qualitatively agree with our results [27], although of course much more work is needed to asses the relevance of our model to actual growth processes. Finally, it is most important to clarify the asymptotic scaling of our model in the high temperature phase. We have obtained that a tensionless surface roughens at a temperature approximately half of that required by a system minimizing surface tension. The morphology at $T>T_{\mathrm{R}}$ is much rougher, at least in the early dynamics, for the former case than for the latter. We believe the asymptotic scaling for $T>T_{\mathrm{R}}, I>0$ is the same in the two cases. If confirmed, this result implies that in realistic systems the linear MBE equation scaling can not be asymptotic. However, the crossover time can be large, presumably observable for appropriate experimental realizations. Work along these lines is in progress [23].

\section{Acknowledgements}

We are indebted to L.A.N. Amaral, A.R. Bishop, J.A. Cuesta, E. Diez, and M. Kotrla for discussions. This work has been supported by CICyT (Spain) grant MAT95-0325. 


\section{References}

[1] A.-L. Barabasi, H.E. Stanley, Fractal Concepts in Surface Growth, Cambridge University Press, Cambridge UK, 1995.

[2] M. Scheffler, R. Zimmermann (Eds.), Proceedings of 23rd International Conference on the Physics of Semiconductors, World Scientific, Berlin, 1996.

[3] S.A. Safran, Statistical Thermodynamics of Surfaces, Interfaces, and Membranes, Addison Wesley, Reading MA, 1994.

[4] M.G. Lagally (Ed.), Kinetics of Ordering and Growth at Surfaces, NATO ASI Series B, Vol. 239, Plenum, New York, 1990, and references therein.

[5] J. Krug, Adv. Phys. (in press).

[6] J. Villain, J. Physique I. 1 (1991) 19.

[7] Z.-W. Lai, S. Das Sarma, Phys. Rev. Lett. 66 (1991) 2348.

[8] C. Herring, J. Appl. Phys. 21 (1950) 301.

[9] W.W. Mullins, J. Appl. Phys. 28 (1957) 333.

[10] H.-N. Yang, Y.-P. Zhao, G.-C. Wang, T.-M. Lu, Phys. Rev. Lett. 76 (1996) 3774.

[11] J.H. Jeffries, J.-K. Zuo, M.M. Craig, ibid. 76 (1996) 4931.

[12] S.T. Chui, J.D. Weeks, Phys. Rev. B. 14 (1976) 4978.

[13] They also showed that any periodic function gives the same static behavior at the roughening point, so there is no loss of generality in choosing $\cos h$.

[14] J.D. Weeks, G.H. Gilmer, Adv. Chem. Phys. 40 (1979) 157.

[15] The discrete sG equation is Eq. (3) with a Laplacian instead of a bi-Laplacian. The corresponding continuum sG equation in the overdamped limit reads $\frac{i h t}{\lambda_{1}}=\nu \nabla^{2} h-\sin h+I(x)+\xi(x, t)$. Here $\nu$ is a surface tension coefficient related to surface stiffness.

[16] F. Falo, A.R. Bishop, P.S. Lomdahl, B. Horovitz, Phys. Rev. B. 43 (1991) 8081.

[17] S.T. Chui, J.D. Weeks, Phys. Rev. Lett. 40 (1978) 733.

[18] A. Sánchez, D. Cai, N. Grønbech-Jensen, A.R. Bishop, Z.J. Wang, Phys. Rev. B. 51 (1995) 14664.

[19] In many MBE situations there is a conserved current of material along the surface, which restricts the deterministic terms in Eq. (4) to be of the form $-\nabla \vec{j}$. This forces the periodic term in the equation to be different from the one considered here, see G. Grinstein, D.-H. Lee, Phys. Rev. Lett. 66 (1991) 177.

[20] L.-H. Tang, T. Natterman, ibid. 66 (1991) 2899.

[21] J. Krug, in Proceedings of the ICTP Workshop on Dynamics of Non-Equilibrium Systems, 1996 (unpublished). Eq. (4) can be thus expected to be relevant in systems for which non-conserving processes are present, and time and length scales are small enough for surface tension effects (see D.D. Vvedensky, A. Zangwill, C.N. Luse, M.R. Wilby, Phys. Rev. E 48 (1993) 852) to be negligible.

[22] P. Noziëres, F. Gallet, J. Physique. 48 (1987) 353.

[23] E. Moro, R. Cuerno, A. Sánchez, in preparation.

[24] That is, a Laplacian term like that in 13 would be generated in the effective equation of motion for a coarse grained system.

[25] The Edwards-Wilkinson equation is the overdamped sG one without the sine term: S.F. Edwards, D.R. Wilkinson, Proc. R. Soc. Lond. A. 381 (1982) 17.

[26] A straightforward proof follows from the deterministic nature of Eq. (3) at $T=0$.

[27] A.S. Arrott, B. Heinrich, S.T. Purcell, 4. 